\title{
L'intégration des nouvelles données probantes dans la pratique et la nécessité de l'application des connaissances
}

\author{
par Scot H Simpson
}

$\mathrm{T}$

out le monde sait que, pour faire des choix, il faut s'appuyer sur des faits probants. Que l'on cherche à choisir un restaurant pour le souper, un film pour la fin de semaine ou les pneus d'hiver les plus sécuritaires pour son véhicule, nous utilisons souvent de l'information, des données probantes, pour guider nos choix. Or, l'importance des données probantes est tout aussi grande dans notre milieu professionnel. Le fait est que les principes fondamentaux des soins fondés sur les données probantes nous encouragent à faire les bons choix cliniques en restant au fait des meilleures données disponibles, en déterminant leur véracité et en les mettant en contexte par l'étude des avantages et des risques, des implications ainsi que des coûts associés au choix ${ }^{1}$. Mais malheureusement, l'application de nouvelles données probantes visant à faciliter les changements dans la pratique n'est pas idéale.

En effet, modifier la pratique clinique est une tâche complexe et exigeante; qui plus est, l'écart notoire entre les données probantes appuyant le changement et les pratiques actuelles nuit à notre capacité d'offrir avec efficacité des soins de qualité à nos patients ${ }^{2-4}$. Produire de nouvelles données probantes à l'aide d'un projet de recherche, présenter les résultats au cours d'un congrès et publier dans une revue, ces actions ne suffisent pas à elles seules à faire évoluer la pratique $e^{5}$. L'on reconnaît plutôt de plus en plus que, pour combler l'écart entre les données et la pratique, il faudra faire appel à un processus appelé " application des connaissances ». Bien qu'il existe plusieurs définitions de ce terme ${ }^{6}$, la plus répandue fait référence à : " un processus dynamique et itératif qui englobe la synthèse, la dissémination, l'échange et l'application conforme à l'éthique des connaissances dans le but d'améliorer la santé, d'offrir de meilleurs produits et services de santé et de renforcer le système de santé ${ }^{7}$ ". On y observe non seulement l'importance accordée à la production et à la diffusion de nouvelles données probantes, mais aussi l'importance centrale accordée à l'application opportune - ou convenable d'un point de vue éthique - des connaissances.

Traditionnellement, les clubs de lecture scientifique et les activités de formation continue servent à diffuser les données probantes et à encourager les praticiens à apporter des changements dans leur pratique. Bien que ces activités puissent aider à faire connaître les nouvelles données probantes, les moyens employés pour présenter l'information ne répondent pas nécessairement aux besoins de tous, ce qui se traduit par une faible mise en application. Constatant la nécessité d'un moyen clair servant d'une part à illustrer comment intégrer les nouveaux résultats de recherche dans la pratique et d'autre part à réduire la confusion progressive entourant la notion d'application des connaissances, Graham et collab. ${ }^{8}$ ont créé le cycle des connaissances à la pratique. Ce cadre conceptuel fait ressortir des facteurs à considérer dans le cheminement s'échelonnant de la création de nouvelles connaissances jusqu'à leur application dans la pratique. Il repose sur sept actions : cerner le problème ainsi que les connaissances devant être appliquées; adapter les connaissances au contexte du milieu; étudier les éléments qui facilitent l'utilisation des connaissances ou qui lui font obstacle; choisir et ajuster la bonne intervention d'application des connaissances; surveiller l'emploi des connaissances; évaluer les résultats; et déterminer des stratégies favorisant le maintien de l'utilisation des connaissances ${ }^{8}$.

Le cadre conceptuel des connaissances à la pratique contient une étape importante : l'évaluation des éléments qui peuvent faciliter l'utilisation des connaissances ou lui faire obstacle ${ }^{2}$. En tant qu'utilisateurs de connaissances, nous sommes déjà conscients de l'importance d'avoir recours à notre sens critique afin d'évaluer les sources de toutes nouvelles informations et de nous assurer de

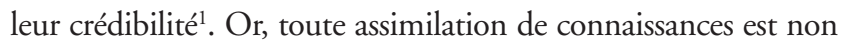


seulement influencée par le contenu et la fiabilité de l'information, mais aussi par le milieu de pratique et par les personnes à qui l'on demande d'intégrer des connaissances dans leur pratique ${ }^{2,8}$. En d'autres termes, la façon de présenter l'information pourrait être tout aussi importante que l'information elle-même. Ainsi, une bonne compréhension du milieu de pratique et des caractéristiques des personnes composant le public cible permettra de créer un message efficace qui facilitera l'application des connaissances.

Dans ce numéro du Journal canadien de la pharmacie hospitalière, Rosenthal et collab. ${ }^{9}$ dressent le portrait d'une initiative servant à déceler les éléments qui peuvent faciliter l'utilisation des connaissances ou lui faire obstacle, et ce, en étudiant le milieu de la pratique de la pharmacie hospitalière d'aujourd'hui. À l'aide d'un sondage Internet destiné aux pharmaciens d'hôpitaux du Canada, ils ont découvert que la plupart des répondants accordent de l'importance aux facteurs de soutien, comme le travail d'équipe et la collaboration, et qu'ils manifestent des caractéristiques axées sur l'accomplissement. Les chercheurs ont aussi relevé que les caractéristiques du milieu et des personnes variaient beaucoup en fonction de la région $\mathrm{du}$ répondant, de son niveau de scolarité, du nombre de ses années de pratique et du temps qu'il passait à effectuer des activités cliniques. Ces différences dans les milieux de pratique et dans les caractéristiques personnelles mettent en relief l'importance pour les gestionnaires des changements de prendre le temps d'approfondir les caractéristiques de leur public cible.

En tant que professionnels de la santé, il est de notre devoir de participer à un apprentissage continu et de toujours chercher de nouvelles informations nous permettant d'offrir des soins de qualité aux patients. Comme nous l'indiquent les principes des soins fondés sur les données probantes, il est normal de rester prudents quant aux nouvelles interventions, car il existe des exemples de «bonnes idées" qui n’en étaient pas vraiment ${ }^{10-12}$. Mais, lorsque nous trouvons des données convaincantes en faveur d'un changement dans notre pratique, nous devons aussi être en mesure de faire part éloquemment de ces informations à nos collègues et de les inviter à revoir leur pratique. Bien que changer sa pratique puisse être un processus lent et exigeant ${ }^{2,5}$, le cycle des connaissances à la pratique offre un cadre conceptuel qui aidera à communiquer efficacement de nouvelles informations et qui en facilitera la mise en pratique ${ }^{8}$. Bref, l'objectif de l'application des connaissances est de réduire l'écart entre les données probantes et la pratique, ce qui permettra d'améliorer les soins aux patients, de renforcer le système de santé et ultimement de faire de nous de meilleurs fournisseurs de soins de santé.

[Traduction par l'éditeur]
Références

1. Guyatt G, Jaeschke R, Wilson MC, Montori VM, Richardson WS. What is evidence-based medicine? In: Guyatt G, Rennie D, Meade MO, Cook DJ, rédacteurs. Users' guides to the medical literature: a manual for evidencebased clinical practice. 3rd ed. New York (NY): McGraw-Hill; 2015. p. 7-14.

2. Grol R, Grimshaw J. From best evidence to best practice: effective implementation of change in patients' care. Lancet. 2003;362(9391):1225-30.

3. Institute of Medicine, Committee on Quality of Health Care in America. Crossing the quality chasm: a new health system for the 21st century. Washington (DC): National Academy Press; 2001. 337 p.

4. Davis D, Evans M, Jadad A, Perrier L, Rath D, Ryan D, et collab. The case for knowledge translation: shortening the journey from evidence to effect. BMJ. 2003;327(7405):33-5.

5. Morris ZS, Wooding S, Grant J. The answer is 17 years, what is the question: understanding time lags in translational research. J R Soc Med. 2011; 104(12):510-20.

6. À propos de l'application des connaissances et de la commercialisation. Ottawa (ON) : Instituts de recherche en santé du Canada; 2014. Publié au : www.cihr-irsc.gc.ca/e/29418.html. Consulté le 15 janvier 2015.

7. Straus SE, Tetroe J, Graham ID. Knowledge translation: what it is and what it isn't. In: Straus SE, Tetroe J, Graham ID, rédacteurs. Knowledge translation in health care: moving from evidence to practice. Oxford (UK): Wiley Blackwell; 2013. p. 3-13.

8. Graham ID, Logan J, Harrison MB, Straus SE, Tetroe J, Caswell W, et collab. Lost in knowledge translation: time for a map? J Contin Educ Health Prof. 2006;26(1):13-24

9. Rosenthal M, Hall KW, Bussières JF, Tsuyuki RT. Professional culture and personality traits of hospital pharmacists across Canada: a fundamental first step in developing effective knowledge translation strategies. Can J Hosp Pharm. 2015;68(2):127-35.

10. Echt DS, Liebson PR, Mitchell LB, Peters RW, Obias-Manno D, Barker $\mathrm{AH}$, et collab. Mortality and morbidity in patients receiving encainide, flecainide, or placebo. The Cardiac Arrhythmia Suppression Trial. N Engl JMed. 1991;324(12):781-8.

11. Grady D, Herrington D, Bittner V, Blumenthal R, Davidson M, Hlatky M, et collab.; HERS Research Group. Cardiovascular disease outcomes during 6.8 years of hormone therapy: Heart and Estrogen/progestin Replacement Study follow-up (HERS II). JAMA. 2002;288(1):49-57.

12. Bosch J, Yusuf S, Gerstein HC, Pogue J, Sheridan P, Dagenais G, et collab. Effect of ramipril on the incidence of diabetes. N Engl J Med. 2006;355(15):1551-62

Scot H Simpson, BSP, Pharm. D., M. Sc., travaille à la Faculté de pharmacie et des sciences pharmaceutiques de I'Université de l'Alberta, à Edmonton, en Alberta. II est aussi rédacteur adjoint du JCHP.

Intérêts concurrents : Aucun déclaré.

Adresse de correspondance :

Dr Scot H Simpson

Faculty of Pharmacy and Pharmaceutical Sciences

3126 Dentistry / Pharmacy

University of Alberta

Edmonton AB T6G 2N8

Courriel : scot@ualberta.ca 